\title{
TO THE BOUNDARY AND BACK-A NUMERICAL STUDY
}

\author{
S. KARNI \\ Department of Mathematics, University of Michigan, Ann Arbor, MI 48109, U.S.A.
}

\begin{abstract}
SUMMARY
This study identifies the key parameters upon which energy absorption at artificial boundaries depends. A thorough numerical study is presented, of typical reflections from open computational boundaries, for problems governed by hyperbolic systems of equations. The emphasis is on systems, where it is often the combination of all boundary procedures that determine the quality of boundary treatment. We study dissipative numerical models which have so far not been analysed to the same extent as non-dissipative models and employ a Lax-Wendroff-type scheme as a prototype. While it is widely accepted that dissipative models tend to give fewer problems than non-dissipative ones, we show a variety of cases where substantial reflections do occur even in 1D and quasi-1D set-ups, where theory predicts best results. This can partly be explained by the vanishing of dissipation in the far field. Group velocity analysis, justifiable on the grounds of weak dissipation, predicts a pathological behaviour which is confirmed by numerical experiments. We demonstrate strong focusing of asymptotic errors generated at the artificial boundary. Internal reflections due to slowly expanding grids are shown for non-linear systems. The need for high-frequency boundary conditions naturally arises and combined low-high-frequency boundary recipes following Higdon, Vichnevetsky and Pariser are adapted to systems and tested. Partial cures are also discussed, mainly in terms of pointing out their theoretically limited potential.
\end{abstract}

KEY WORDS Hyperbolic systems Artificial boundary Dissipative Group velocity

\section{INTRODUCTION}

In many CFD applications it is required to solve problems in unbounded physical domains. Numerical solutions, by their nature, cannot be obtained on infinite domains and the problem has first to be converted to a finite region. It is customary to truncate the domain by introducing an artificial boundary at some arbitrary finite distance. Well-posedness of the truncated problem usually requires boundary conditions (BCs) at the artificial boundary, which should ideally allow the outgoing waves to pass through without generating reflections. Far-field reflections are one of several mechanisms that effect the quality of solution. Indeed, reflections not only degrade time accuracy of transient solutions but also inhibit their convergence to steady states. In extreme cases they may render the solution unstable. Moreover, high energy absorption at the boundaries usually enables the bringing in of the outer boundaries, thereby reducing computational costs. Practical computations, however, are often much too envolved to pinpoint the precise way in which the BCs come into paly, and their effect usually gets absorbed and lost in the overall complexity of the calculation alongside other factors such as the numerical algorithm, geometrical complexity, artificial viscosity, etc. Whatever effect they may have on the solution in the interior, it is common wisdom that moving the boundaries further out will reduce it. This raises other complications such as large and usually highly stretched grids on which formal accuracy is often 
lost and which slow down convergence to steady states. Better understanding of far-field reflection effects on the solution requires one to study the problem in isolation, by looking at model problems stripped of other computational complexities.

The theory of characteristics provides a general framework for the imposition of absorbing BCs. For one-dimensional problems the theory leaves the freedom to specify as many BCs as there are incoming characteristics at the boundary. The outgoing part of the solution is predetermined by the initial data and is accordingly extrapolated from the interior of the domain. In multidimensional problems wave propagation is a lot more complex. One-dimensional characteristic analysis is still accepted as a general guideline but is no longer fully justifiable. Common absorbing boundary recipes often resort to $1 \mathrm{D}$ arguments normal to the boundary, which are strictly valid for waves travelling in that direction only. Asymptotic expansions lead to higher-order boundary operators which account for angular dependence. ${ }^{1}$ Other asymptotic arguments lead to boundary operators which become increasingly more accurate as the boundary approaches infinity. ${ }^{2}$ To accomplish high absorption at artificial boundaries, the outgoing part of the solution is identified and the extrapolation procedures are made to match its propagation pattern. ${ }^{1-4}$ These procedures can broadly be divided into three categories and give rise to various sources of errors. An analytic BC based on an asymptotic argument generates errors of the order of the neglected terms. When replaced by a numerical approximation, further discretization errors are introduced. In the direct numerical approach the boundary operator is matched to the outgoing part of the numerical solution, usually to a particular (low) wave frequency. ${ }^{5,6}$ Asymptotic expansions then determine its efficiency in absorbing neighbouring frequencies. This approach has the advantage that it can be targeted at absorbing non-physical parasitic modes which are 'beyond the reach' of any analytic approach, ${ }^{5,7}$ but is in general very sensitive to local numerical parameters. In the third and probably largest class are empirical BCs guided by practical experience. These many times prove at least as effective as the more theoretically sound BCs in the previous two classes. ${ }^{8,9}$ To illuminate boundary reflection mechanisms, we have chosen to concentrate on $1 \mathrm{D}$ and quasi1D test problems, cases where theory predicts best results. We show that even in these 'best' cases, widely held as easy cases, strong reflections may and do occur. Asymptotic boundary errors due to genuine multidimensionality are, on the whole, better appreciated and will tend to amplify the reflection mechanisms discussed in this work. We do not, however, feel that considering more involved problems will shed more light on the present study. On the contrary, inevitable additional complexities which are somewhat irrelevant to the present study will tend to obscure its simplicity and clarity.

Discrete wave propagation in non-dissipative models has been analysed by various authors. ${ }^{5,10-12}$ Yet, many flow simulations use dissipative models which are widely accepted as 'better behaved' and relatively problem-free (e.g. stability problems near shocks or near other boundaries). In this work we concentrate on the less studied class of dissipative models and use the Lax-Wendroff (LW) scheme as a popular prototype. In view of their popularity, it is of particular importance to realize that even dissipative models may lead to substantial far-field reflections, which, although classified as high-order effects, may reach intolerable levels. We show that this can partly be explained by the vanishing of dissipation in the far field. Group velocity analysis, though strictly applicable to non-dissipative models, ${ }^{13}$ becomes justifiable on the grounds of weak dissipation. ${ }^{10.14}$ It predicts a pathological behaviour known to exist with several non-dissipative models $^{10-12,15}$ and which tends to amplify far-field reflections. We identify the key numerical parameters upon which energy absorption at artificial boundaries depends and pinpoint the way in which they come into play. Our emphasis is on systems where it is often the combination of interior scheme and all boundary procedures that determines the quality of boundary treatment. We confirm the theoretical predictions by a thorough numerical investigation. We demonstrate 
strong focusing of asymptotic errors generated at the artificial boundary. Internal reflections due to slowly varying grids are also shown. The need for high-frequency boundary recipes naturally arises and combined low-high-frequency BCs following Higdon ${ }^{5}$ and Vichnevetsky and Pariser ${ }^{7}$ are adapted to systems and tested. Partial cures are also discussed, mainly in terms of pointing out their theoretically limited potential.

Finally, we mention that the pathological behaviour of the LW scheme as predicted by group velocity analysis is in fact not unique to this scheme. It applies to a much wider class of dissipative symmetric schemes of optimal accuracy and to symmetric Runge-Kutta-type schemes. ${ }^{16}$ Upwind schemes, in this context, live up to their good reputation. Their inherent dissipation, although greatly reduced in the far field, is still sufficient to damp out the reflected waves. ${ }^{16}$

\section{DISCRETE WAVE PROPAGATION}

\section{Discrete model, finite representation and spurious modes}

As a traditional model equation, consider the advection equation

$$
u_{t}+a u_{x}=0 \text {. }
$$

Although (1) is non-dispersive, discrete numerical models to approximate (1) always are. Depending on the numerical model, different wave frequencies will propagate at different speeds, causing the numerical solution to disperse and distort. While dispersion errors of well-resolved low frequencies are negligible, they become quite large for poorly resolved high frequency waves, leading to totally spurious non-physical behaviour.

Let a grid be defined by $x_{j}=j h, t_{n}=n k$, where $h$ and $k$ are the grid spacings in the $x$ - and $t$-directions. The discrete representation of a Fourier mode of wave number $\xi$,

$$
f(x)=f(j h)=\mathrm{e}^{i \xi j h}=\left(\mathrm{e}^{i \xi h}\right)^{j},
$$

is determined by the parameter $\xi h$, which assumes values in the range $(-\pi, \pi]$. A one-dimensional grid with $N$ points admits $N$ modes, the highest possible frequency of which corresponds to $\xi h=\pi$ and is of saw-tooth character. Since far-field flows tend to be smooth and slowly varying, computational grids often become increasingly coarser and sampling points become far apart. Consequently, an increasing number of modes tends to appear as high frequencies relative to the grid and dispersion errors become large and more acute. The main mechanism that can rescue the solution from being contaminated by large dispersion errors is dissipation. Unfortunately, the inherent dissipation of many schemes tends to diminish under far-field conditions as a result of the occurrence of small CFL numbers.

Let (1) be approximated by the LW scheme

$$
u_{j}^{n+1}=u_{j}^{n}-\frac{v}{2}\left(u_{j+1}^{n}-u_{j-1}^{n}\right)+\frac{v^{2}}{2}\left(u_{j-1}^{n}-2 u_{j}^{n}+u_{j+1}^{n}\right),
$$

with $v=a k / h$ the CFL number. The discrete dispersion relation of (3) is obtained by substituting a travelling wave solution $u_{j}^{n}=\exp (i \omega n k-\mathrm{i} \xi j h)$ and reads

$$
\mathrm{e}^{\mathrm{i} \omega k}=1+\mathrm{i} v \sin (\xi h)+v^{2}[\cos (\xi h)-1] .
$$

It is non-linear complex relation between $\omega$ and $\xi$ corresponding to (3) being a dispersive and dissipative numerical model. Equation (4) is a quadratic relation between $Z=\mathrm{e}^{\mathrm{i} \omega \boldsymbol{k}}$ and $\kappa=\mathrm{e}^{\mathrm{i} \xi \boldsymbol{h}}$ :

$$
Z=1-\frac{v}{2}\left(\kappa^{-1}-\kappa\right)+\frac{v^{2}}{2}\left(\kappa^{-1}-2+\kappa\right) \text {. }
$$


Any fixed frequency $Z_{0}=Z\left(\omega_{0}\right)$ admits two spatial modes $\kappa^{ \pm}$,

$$
\kappa^{ \pm}=\frac{1}{v(v+1)}\left(v^{2}+Z_{0}-1 \pm \Delta\right), \quad \Delta^{2}=\left(1-Z_{0}\right)^{2}+v^{2}\left(2 Z_{0}-1\right)
$$

which correspond to two distinct wave numbers $\xi^{ \pm}$. Only one of these wave numbers corresponds to a physically admissible mode. The other is an inevitable numerical by-product, which is a direct consequence of approximating the $x$-derivative term in (1) using three grid points. The admittance of spurious modes in high-order numerical models has mainly been recognized in non-dissipative models. Their occurrence in dissipative models is not generally regarded as troublesome because they do not usually trigger instabilities. Yet, while stability is a crucial prerequisite for a numerical solution to be possible, it is by no means an only requirement. Our numerical study shows that the admittance of spurious modes in dissipative models should not be dismissed too lightly, since it raises other severe computational problems, e.g. degrades time accuracy of transient solutions and inhibits their convergence to stady states.

Taking the steady state limit $\omega k \rightarrow 0(Z \rightarrow 1)$,

$$
\kappa^{+} \rightarrow 1, \quad \kappa^{-} \rightarrow \frac{v-1}{v+1}
$$

reveals that $\kappa^{+}$is the physically consistent mode while $\kappa^{-}$is the spurious one and is of saw-tooth character since $|v| \leq 1$ for stability.

Let $\xi \in \mathbb{R}$; then $\omega=\omega_{\mathbb{R}}+i \omega_{1} \in \mathbb{C}$ is complex and (4) can be separated into its real and imaginary parts

$$
\mathrm{e}^{-\omega_{\mathbf{I}} k} \sin \left(\omega_{\mathrm{R}} k\right)=v \sin (\xi h), \quad \mathrm{e}^{-\omega_{\mathbf{I}} k} \cos \left(\omega_{\mathrm{R}} k\right)=1+v^{2}[\cos (\xi h)-1] .
$$

Eliminating $\omega_{1}$ in (8) yields

$$
\tan \left(\omega_{\mathbf{R}} k\right)=\frac{v \sin (\xi h)}{1+v^{2}[\cos (\xi h)-1]} .
$$

In Figure $1, \omega_{1} k$ and $\omega_{\mathrm{R}} k$ are plotted against $\xi h$ for different values of $v$. For $v \ll 1, \omega_{1} k \simeq 0$ over the whole range of $\xi h$. In fact, dissipation is maximal when $\xi h=\pi$, yielding the estimate

$$
\omega_{1} k \leq \max \left(\omega_{1} k\right)=-\ln \left(1-2 v^{2}\right) \simeq 2 v^{2} .
$$

That is, for sufficiently small CFL numbers, dissipation is very weak and (9) may replace (4) as a dispersions relation.

\section{Group velocity}

The way dispersion errors manifest themselves in discrete wave propagation is best revealed by using the concept of group velocity, first introduced into discrete model analysis by Trefethen. ${ }^{10,14}$ Although strictly applicable in the non-dissipative case, ${ }^{13}$ the analysis still yields good predictions if dissipation is weak. It can thus be justifiably used to analyse far-field wave propagation (see (10)). Implicit differentiation of (9) yields the approximate group velocity

$$
C_{\mathrm{g}} \simeq \frac{\mathrm{d} \omega_{\mathrm{R}}}{\mathrm{d} \xi}=\frac{a \cos (\xi h)+a v^{2}[1-\cos (\xi h)]}{\left\{1-v^{2}[1-\cos (\xi h)]\right\}^{2}+v^{2} \sin ^{2}(\xi h)},
$$

with the understanding that (11) is valid only for $|v| \ll 1$. Figure 2 shows $C_{\mathrm{g}} / a$ as a function of $\xi h$ for various CFL numbers. We observe that in the extreme cases

$$
C_{\mathrm{B}}(\xi h=0)=a, \quad C_{\mathrm{g}}(\xi h=\pi)=-a /\left(1-2 v^{2}\right),
$$




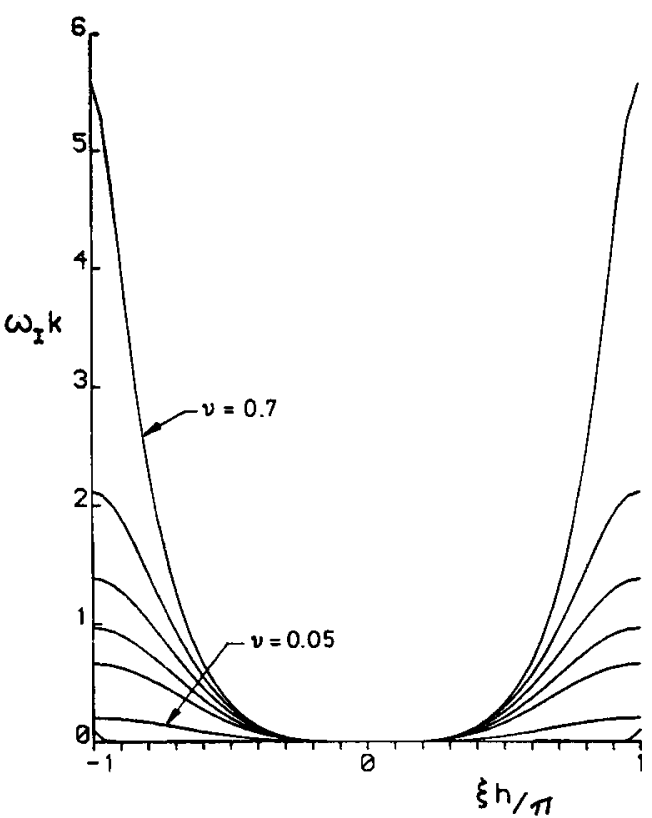

(a)

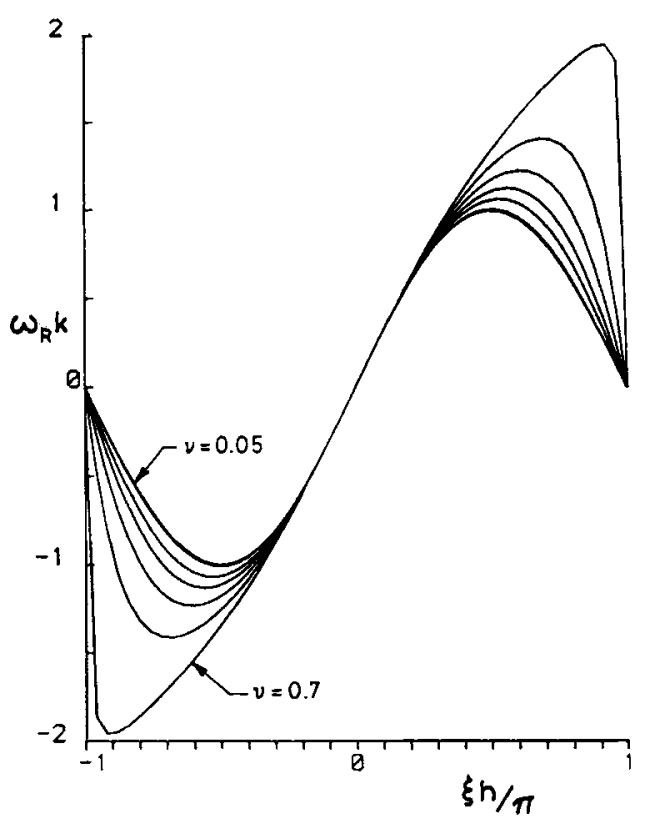

(b)

Figure 1. Imaginary and real parts of dispersion relation of equation (4) for various CFL numbers: (a) $\omega_{1} k$ and (b) $\omega_{\mathrm{R}} k$ against $\xi h$

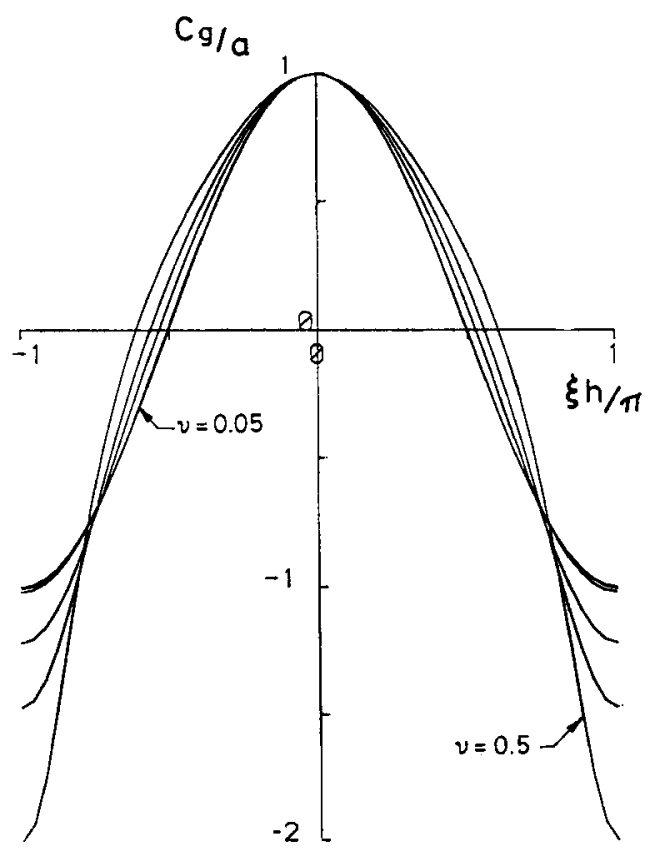

Figure 2. Approximate group velocity $C_{\mathrm{a}} / a$ as a function of $\xi h$ for various $\mathrm{CFL}$ numbers 
suggesting that well-resolved smooth waves propagate at the physically correct speed while poorly resolved waves not only travel with the wrong speed but may also move in the wrong direction if $v^{2}<\frac{1}{2}$. The damping coefficient for the mode $\xi h=\pi$ is, by (5),

$$
Z(\xi h=\pi)=1-2 v^{2}
$$

and tends to unity in the limit $|v| \ll 1$. Since at small CFL numbers waves propagate through fewer grid cells, the full implication of almost undamped waves moving in the physically wrong direction has to be assessed after a fixed time $T$ rather than after a fixed number of time steps, $N$, i.e. in the double limit $|v| \rightarrow 0, N \rightarrow \infty$, such that $N k=T$ is fixed. Assuming $h=1$ and $a=1$, the accumulative damping effect is

$$
Z^{N}=Z^{T / v}=\left(1-2 v^{2}\right)^{T / v} \simeq \mathrm{e}^{-2 v T} \underset{|v| \ll 1}{\longrightarrow} 1,
$$

implying that in the limit of small CFL numbers, spurious modes are not dissipated at all. This pathological behaviour is not unique to the LW scheme. It applies to a much wider class of dissipative schemes, including symmetric schemes of optimal accuracy and Runge-Kutta-type schemes. ${ }^{16}$ It is also known to exist with several non-dissipative models. ${ }^{10-12,15}$ Upwind schemes, in that context, live up to their good reputation. Their inherent dissipation, although greatly reduced in the far field, is still sufficient to damp spurious modes. ${ }^{16}$ In particular, the accumulative damping coefficient in the dissipative upwind case is of the form

$$
Z^{N}=(1-c v)^{T / v} \underset{|v| \ll 1}{\longrightarrow} \mathrm{e}^{-c T}
$$

for some positive scheme-dependent constant $c$, i.e. the waves are still dissipated.

Far-field conditions thus combine in a very unfavourable manner. On the one hand, an increasing number of modes tend to appear as high frequencies and induce large dispersion errors. On the other hand, local CFL numbers are small, dissipation diminishes and these spurious modes tend to persist.

\section{NUMERICAL EXPERIMENTS}

\section{Governing equations}

In the following, $\rho, u, e, p, c$ and $\gamma$ represent the density, velocity, total energy, pressure, sound speed and specific heat ratio respectively. Two systems of equations were tested.

The ID unsteady Euler equations

$$
\left(\begin{array}{c}
\rho \\
\rho u \\
e
\end{array}\right)_{t}+\left(\begin{array}{c}
\rho u \\
\rho u^{2}+p \\
u e+u p
\end{array}\right)_{x}=0
$$

Using the ideal gas assumption, $p$ is found from

$$
e=\frac{p}{\gamma-1}+\frac{1}{2} \rho u^{2}
$$

Linear combinations of the equations in (12) yield the characteristic equations ${ }^{13}$

$$
\begin{aligned}
\mathrm{d} p-\rho c \mathrm{~d} u=0 & \text { on } \mathrm{d} x / \mathrm{d} t=u-c \\
\mathrm{~d} p-c^{2} \mathrm{~d} \rho=0 & \text { on } \mathrm{d} x / \mathrm{d} t=u \\
\mathrm{~d} p+\rho c \mathrm{~d} u=0 & \text { on } \mathrm{d} x / \mathrm{d} t=u+c
\end{aligned}
$$


which are later referred to as $(u-c),(u)$ and $(u+c)$ waves respectively.

The $2 D$ unsteady linearized Euler equations with axial symmetry

$$
\left(\begin{array}{l}
p \\
q
\end{array}\right)_{t}+\left(\begin{array}{ll}
0 & 1 \\
1 & 0
\end{array}\right)\left(\begin{array}{l}
p \\
q
\end{array}\right)_{r}+\left(\begin{array}{c}
q / r \\
0
\end{array}\right)=0
$$

The linearization is relative to a state of rest and $\rho=1, c=1$, with $q=u \cos \vartheta+v \sin \vartheta$ the radial speed. Adding and subtracting the equations in (14) yields the characteristic equations

$$
\begin{array}{ll}
\mathrm{d} p-\mathrm{d} q-\int(q / r) \mathrm{d} r=0 & \text { on } \mathrm{d} r / \mathrm{d} t=-1, \\
\mathrm{~d} p+\mathrm{d} q+\int(q / r) \mathrm{d} r=0 & \text { on } \mathrm{d} r / \mathrm{d} t=1,
\end{array}
$$

which are later referred to as $(p-q)$ and $(p+q)$ waves respectively.

\section{Boundary conditions}

In all tests the far-field boundary is subsonic. Five BCs were tested.

(i) Specify pressure $p=p_{\infty}$ and extrapolate remaining quantities using outgoing characteristic equations.

(ii) Specify zero incoming Riemann invariant and extrapolate remaining quantities using outgoing characteristic equations. ${ }^{3}$

(iii) Impose Roe's conditions to treat the acoustic waves. ${ }^{4}$

(iv) Overspecify-specify all quantities.

(v) Underspecify-zero-order extrapolate all quantities.

We make the following observations.

(a) Various variants of (i) where $p=p_{\infty}$ is either explicitly specified or implicitly implied in the steady state limit are in wide use (e.g. in aeronautical flow calculations ${ }^{2,8,9}$ ). They are all motivated by the fact that in external aerodynamic flows the far-field pressure tends to its undisturbed value $p_{\infty}$. However, pressure equals $p_{\infty}$ only at infinity itself, hence BC (i) is only approximate. By placing the boundaries at sufficiently large distance, it is hoped that $p$ at the boundary is not far off $p_{\infty}$ and that the resulting inaccuracies will remain confined to the vicinity of the remote boundary. How far the boundary has to be moved before this effect becomes acceptable remains to be investigated numerically.

(b) BCs (ii) are probably still the most widely used conditions in external aeronautical applications, either in their non-linear version (13) or in their linear version. ${ }^{17}$ Being based on $1 D$ arguments, they are expected to absorb best waves travelling normal to the boundary. Asymptotic expansions are required for waves travelling in other directions. ${ }^{1}$

(c) The gradient form of BCs (ii), (iii) and (v) implies that converged steady states are datadependent, hence weak limits. A correction term is sometimes added to drive the steady state farfield pressure towards its correct asymptotic value. ${ }^{8}$

(d) Both over- and underspecification of the boundary yield ill-posed problems. Underspecification is also formally unstable. ${ }^{18}$ They were included because in many flow calculations the boundary type is either not known a priori or it changes its type in a somewhat arbitrary manner during the transient phase of the calculation. For that reason, the boundary type is many times predetermined by a crude rule of thumb and over- and underspecifications may and do occur. It is interesting to see that in many circumstances they seem to perform no worse than other, betterposed BCs. 


\section{Numerical tests}

Test A (Figure 3). The governing equations are (12). A high pressure fed through the left-hand boundary (LHB) generates a right-moving moderate shock followed by a contact discontinuity propagating into a uniform initial state. The waves are expected to be absorbed by the RHB. The method of solution is Roe's field decomposition with the LW scheme applied to each characteristic field. ${ }^{19}$ No flux limiter is used. The test has been conducted on a uniform grid with $k / h=0.5$ and 0.05 and on a mildly expanding grid with $h_{j+1} / h_{j}=1.05$. An adaptation of the LW scheme to nonuniform grids, which is conservative and retains second-order accuracy on the above mesh, is given in Reference 20. Figure 3 shows the results obtained with BCs (ii). It is observed that small CFL numbers greatly reduce the absorption at the boundary. Very mild grid stretching causes wave propagation to become much more oscillatory and is sufficient to generate substantial boundary reflections of a saw-tooth character. These typical numerical reflections can be confirmed as $(u)$ waves moving in the wrong direction, as admitted by the governing $L W$ scheme. Since dissipation is weak, the reflected waves persist and corrupt the consistent physical solution. Similar results were observed with BCs (iii) and (v).

Test $B$ (Figure 4). The governing equations are (12). The grid stretches from the centre in both directions at a rate of $2 \%$. Initial data correspond to a $(u)$ wave and to a $(u-c)$ wave respectively (see (13)). The initial wave, while propagating in the correct direction, gradually penetrates the coarse part of the grid. At some critical point the local wave number becomes such that the group velocity changes sign (for small CFL numbers this corresponds to a wavelength $\lambda \simeq 4 h$ ). The wave reflects internally and propagates as a saw-tooth wave until it reaches the coarse region, where it reflects again and the low frequency is recovered. This trapped wave continues to bounce back and forth until it dissipates. In computations which march in time towards the steady state limit, internal reflections can be quite troublesome since they inhibit convergence to steady state.

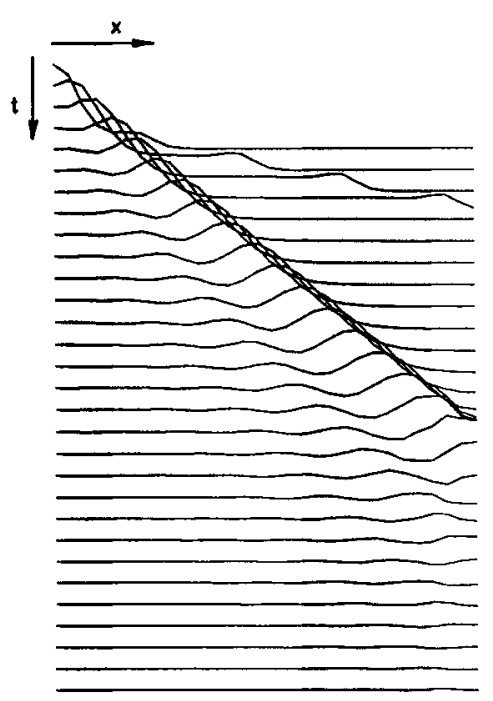

(a)

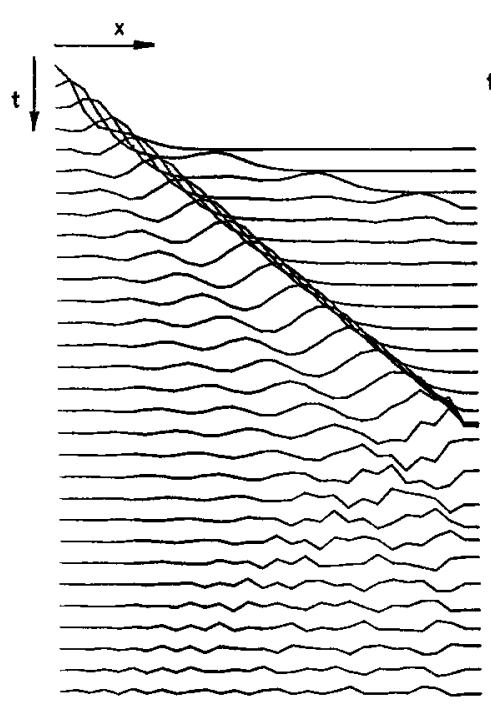

(b)

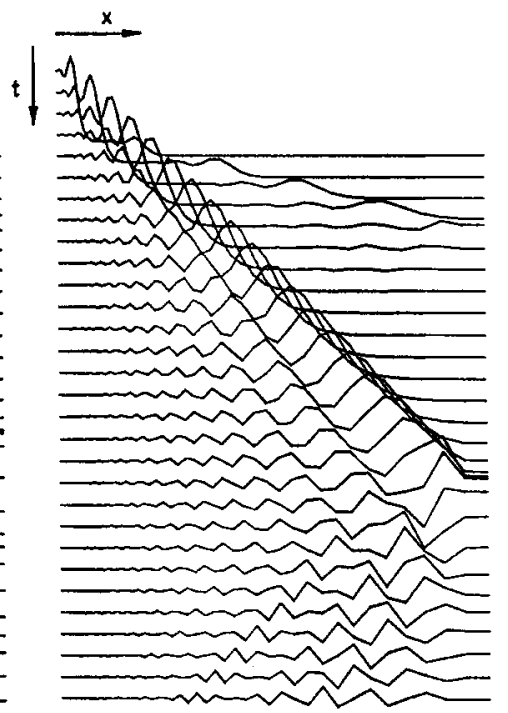

(c)

Figure 3. 1D Euler equations-evolution of density profiles with BC (ii); (a) $k / h=0.5$; (b) $k / h=0.05 ;$ (c) $h_{j+1} / h_{j}=1.05$ 


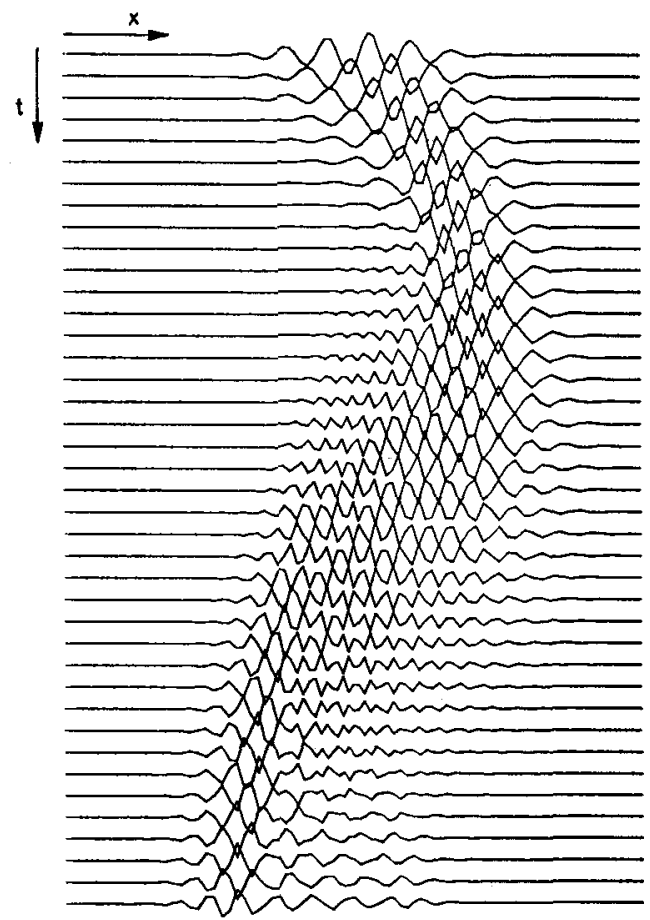

(a)

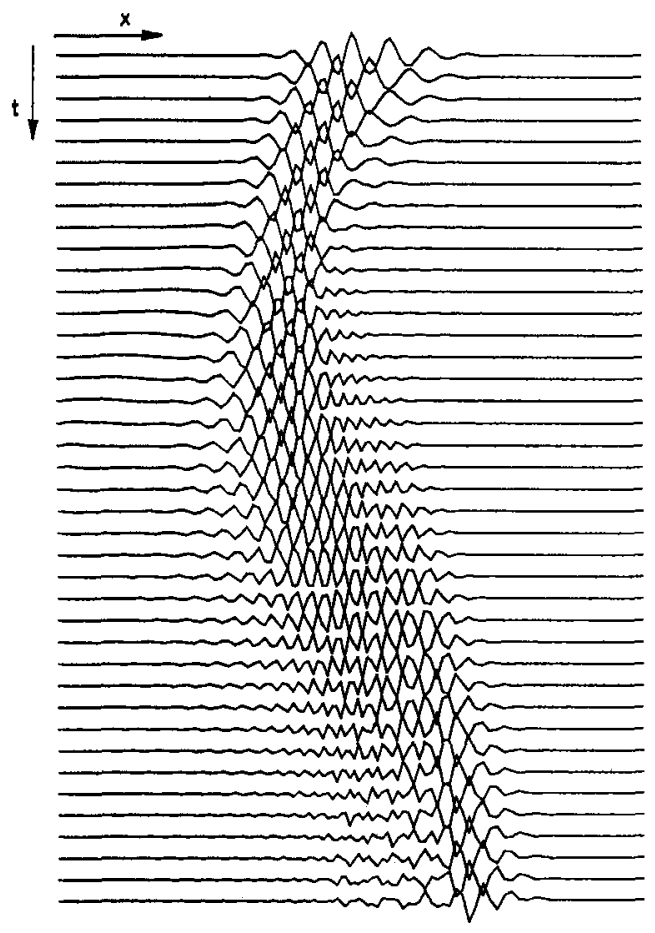

(b)

Figure 4. 1D Euler equations - internal reflections of simple waves on a $2 \%$ expanding grid, evolution of density profiles: (a) (u) wave; (b) $(u-c)$ wave

Trapped waves under non-dissipative schemes have been discussed and studied. ${ }^{11,12}$ Analysis suggests and experiments confirm that in far-field conditions, symmetric dissipative schemes behave in much the same way.

Test $C$ (Figure 5). The governing equations are (14). Initial data correspond to a pressure hump $q=0, p=1+\exp \left(-\sigma r^{2}\right)$, which is expected to decay as it moves away from the origin. The free boundary solution is $(p, q)=(1,0)$. The method of solution is the same as in Test A with both flux- and source-like terms projected onto the characteristic fields. Figure 5 shows the evolution with time of pressure profiles with BC (i) applied at the RHB. Although $p=p_{\infty}$ is not far off the true pressure value at the boundary, this small discrepancy is sufficient to generate a weak reflected wave which bounces off the boundary and then focuses very strongly as it converges towards the origin, reaching intolerable error at the origin itself. The wave continues to bounce back and forth and requires a good number of 'round trips' before the solution converges. This is summarized Tables I and II. In this example, owing to the symmetry of the problem, the focusing effect is particularly strong, becoming even more pronounced in a $3 D$ set-up (Figure $5(b)$ ). A similar behaviour, albeit in a less pronounced manner, is expected to occur whenever $p=p_{\infty}$ is specified. BCs (ii)-(v) completely absorb the wave, including (iv) which may be regarded as a stricter version of $\mathrm{BC}(\mathrm{i})$.

Test $D$ (Figure 6). The governing equations are (14). Initial conditions are a high-frequency wave packet $q=0, p=1+\exp \left[-\sigma\left(r-r_{0}\right)^{2}\right] \cos (\pi r)$, which is expected to break into two wave 


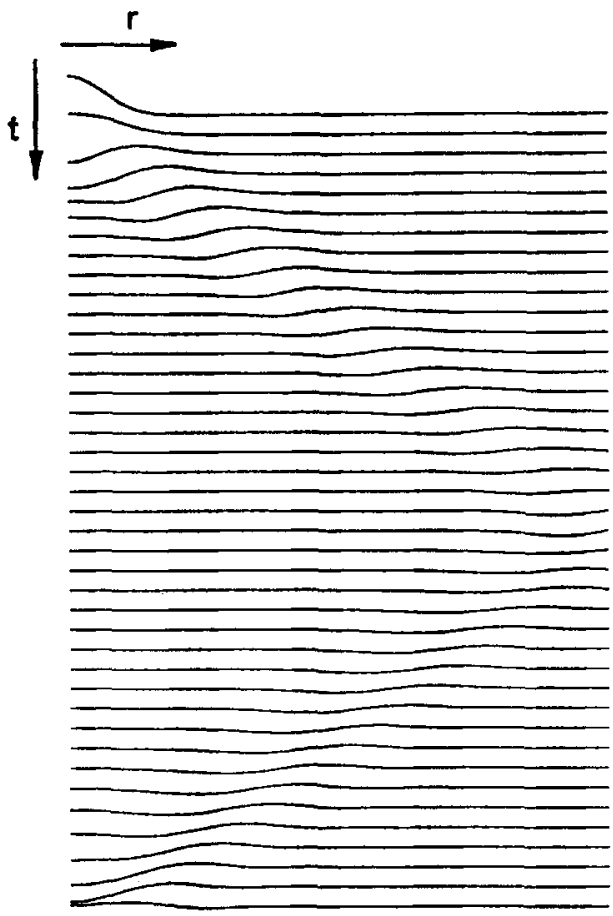

(a)

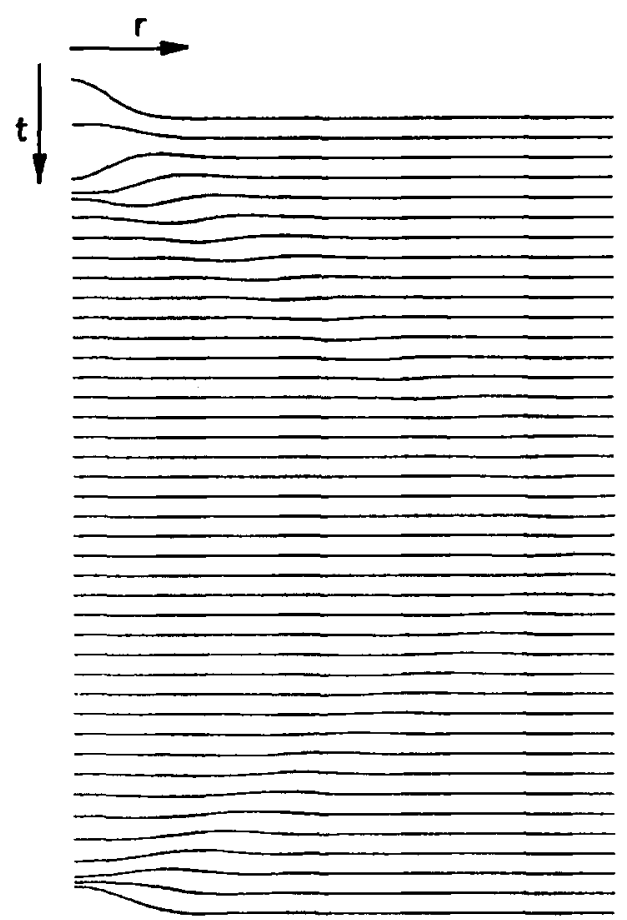

(b)

Figure 5. Partial reflection and strong focusing of a pressure wave with BC (i): (a) 2D and (b) 3D linearized Euler equations with radial symmetry

Table I. Focusing of pressure wave against boundary distance

\begin{tabular}{lcc}
\hline $\begin{array}{l}\text { No. of grid } \\
\text { points }\end{array}$ & $\begin{array}{c}\text { Signal amplitude } \\
\text { near the RHB }\end{array}$ & $\begin{array}{c}\text { Maximum reflected } \\
\text { amplitude at the origin }\end{array}$ \\
\hline 30 & 1.080 & 1.627 \\
50 & 1.051 & 1.500 \\
100 & 1.023 & 1.357 \\
200 & 1.013 & 1.247 \\
\hline
\end{tabular}

Table II. Maximal reflected pressure amplitude at the origin for successive reflections

\begin{tabular}{lcccc}
\hline $\begin{array}{l}\text { No of grid } \\
\text { points }\end{array}$ & $\begin{array}{c}\text { First } \\
\text { focusing }\end{array}$ & Second & Third & Fourth \\
\hline 30 & 1.627 & 1.511 & 1.416 & 1.381 \\
50 & 1.500 & 1.396 & 1.320 & 1.276 \\
100 & 1.357 & 1.274 & 1.220 & 1.189 \\
200 & 1.247 & 1.185 & 1.142 & - \\
\hline
\end{tabular}



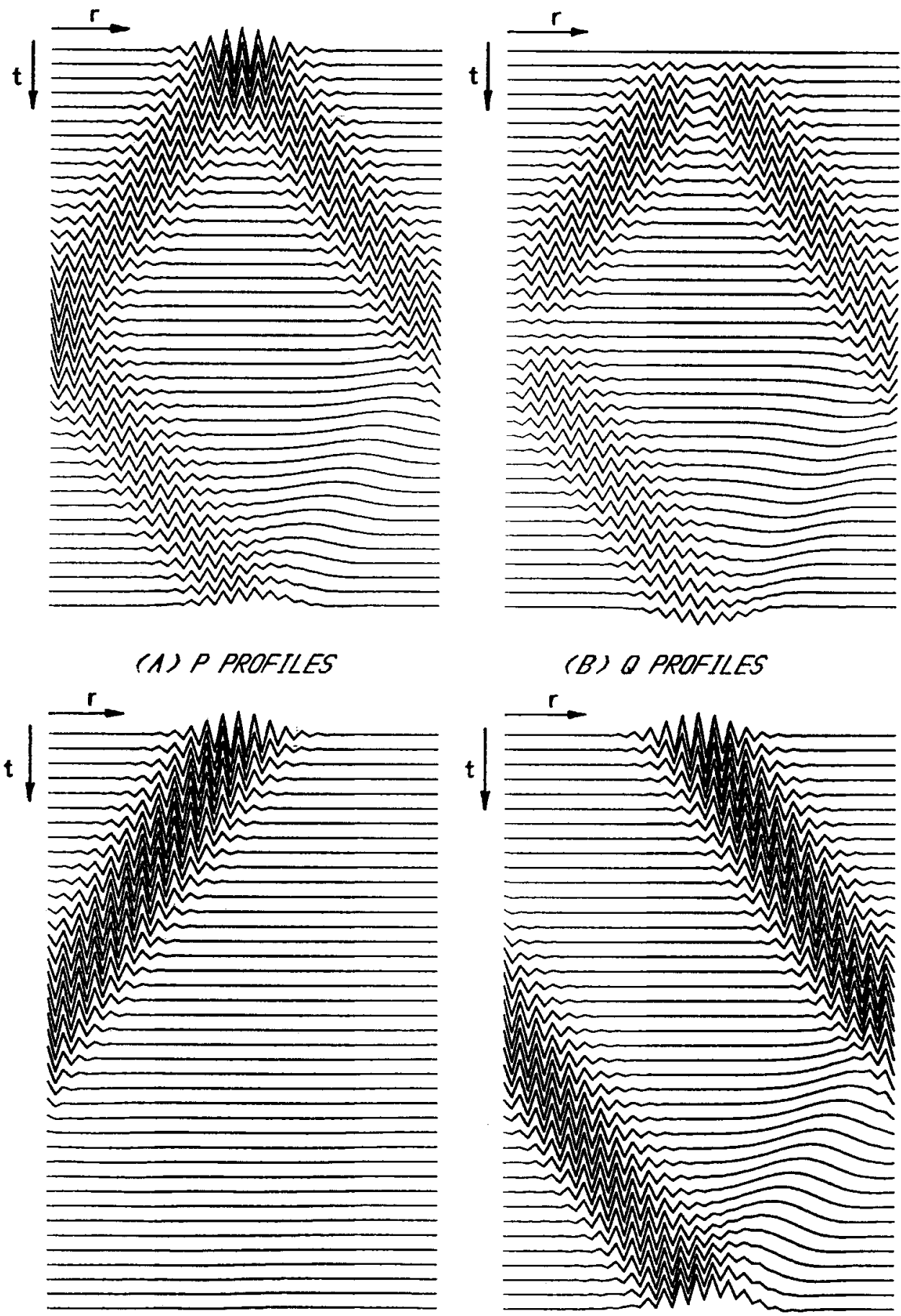

\section{(B) Q PROFILES}

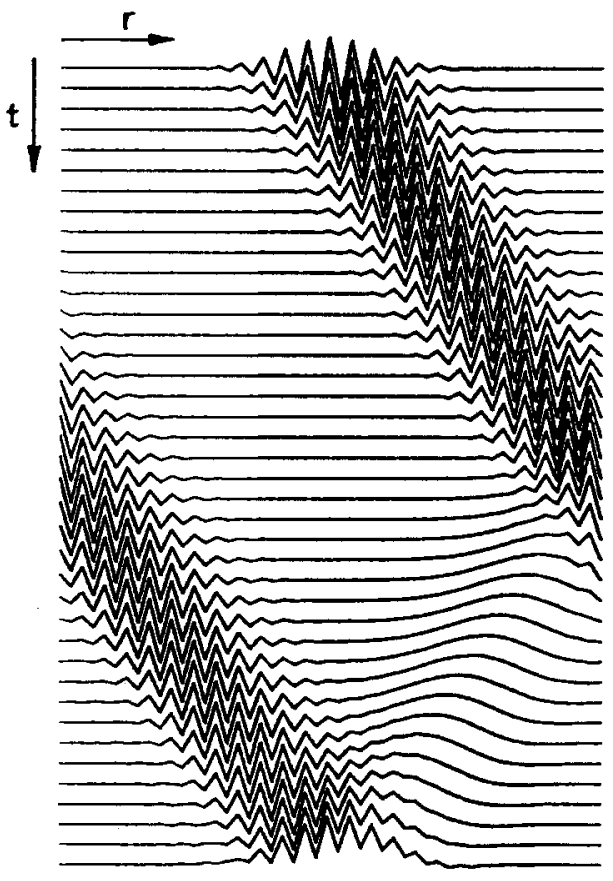

(C) $P+O$ PROFILES

(D) P-O PROFILES

Figure 6. Axisymmetric linearized Euler equations-high-frequency pressure wave reflected from an artificial boundary with BCs (ii) 
packets of half the original strength moving in opposite directions. At first sight this is what appears to happen in Figure 6. Yet, simple wave plots reveal that both wave packets are moving 'backwards'. The result is that the designated left-running $(p-q)$ wave reaches the RHB. The BCs are not designed to absorb waves of the opposite family and reflect the oncoming wave. While saw-tooth waves are easily recognizable as numerical noise, the reflected waves are smooth and much more harmful since they can no longer be distinguished from consistent solutions. BCs (iii) and (v) displayed similar behaviour.

\section{High-frequency $B C s$}

The direct numerical approach enables the design of BCs specially targeted at absorbing high frequencies. Here we follow a procedure suggested by Higdon. ${ }^{5}$ Consider a general boundary operator of the form

$$
B\left(K^{-1}, \mathbb{Z}^{-1}\right) u_{J \max }^{n+1}=0,
$$

where $\mathbb{K}$ and $\mathbb{Z}$ are the shift operators $\mathbb{K} u_{j}^{n}=u_{j+1}^{n}$ and $\mathbb{Z} u_{j}^{n}=u_{j}^{n+1}$ and $B$ is a polynomial in these operators. If an admissible discrete wave of the form $u_{j}^{n}=Z_{0}^{n} \kappa_{0}^{j}$ is to be absorbed by (16), then $B\left(\kappa_{0}^{-1}, Z_{0}^{-1}\right)=0$. For the saw-tooth wave $\kappa_{0}=-1$, and by (5), $Z_{0}=1-2 v^{2} \simeq 1$ for $|v| \ll 1$. The desired boundary operator must have $(\kappa, Z)=(-1,1)$ as a root. Not all such BCs are stable, but it can be shown that

$$
B\left(\mathbb{K}^{-1}, \mathbb{Z}^{-1}\right) u_{J_{\max }^{n+1}}^{n}=0, \quad B=\left\{I-\frac{I+\mathbb{Z}^{-1}}{2} \frac{I-\mathbb{K}^{-1}}{2}\right\}
$$

is stable. ${ }^{5}$ We have adapted (17) to systems of equations in two ways:

(a) To the Riemann invariants in (ii) neglecting the non-homogeneous term near the far-field boundary,

$$
B\left(\mathbb{K}^{-1}, \mathbb{Z}^{-1}\right)(p-q)_{J \max }^{n+1}=0, \quad B\left(\mathbb{K}, \mathbb{Z}^{-1}\right)(p+q)_{J \max }^{n+1}=0 ;
$$

(b) To Roe's BCs (iii) neglecting the non-homogeneous terms,

$$
B\left(\mathbb{K}^{-1}, \mathbb{Z}^{-1}\right) p_{J \max }^{n+1}=0, \quad B\left(\mathbb{K}^{-1}, \mathbb{Z}^{-1}\right) q_{J \max }^{n+1}=0 .
$$

Since $\left(\kappa_{0}, Z_{0}\right)=(1,1)$ is bounded away from zero in $(17)$, low frequencies cannot be absorbed by this procedure. This is confirmed in Figure 7, which was obtained by the boundary operators in (18). Similar results were obtained by (19).

Combined BCs designed to absorb both low- and high-frequency waves are adapted to systems in the following way:

$$
\left\{I-\frac{I+\mathbb{Z}^{-1}}{2} \frac{I-\mathbb{K}^{-1}}{2}\right\}(p-q)_{J \max }^{n+1}=0, \quad\left\{I-\frac{I+\mathbb{Z}^{-1}}{2} \frac{I+\mathbb{K}^{-1}}{2}\right\}(p+q)_{J \max }^{n+1}=0 .
$$

$\mathrm{BCs}(20)$ are used in Figure 8 and prove effective for both ranges of frequency. The very weak reflections observed in Figure 8 are due to the sensitivity of BCs (20) to local wave numbers. Another high-frequency BC suggested in Reference 7 was combined with a standard first-order extrapolation procedure and adapted to systems as follows:

$$
\left\{I+\mathbb{K}^{-1}\right\}(p-q)_{J \max }^{n+1}=0, \quad\left\{I-\mathbb{Z}^{-1}\left[v \mathbb{K}^{-1}+(1-v) I\right]\right\}(p+q)_{J \max }^{n+1}=0 .
$$

It gave satisfactory absorption of high frequencies but partly reflected low frequencies, probably because the first-order extrapolation procedure is too crude. 


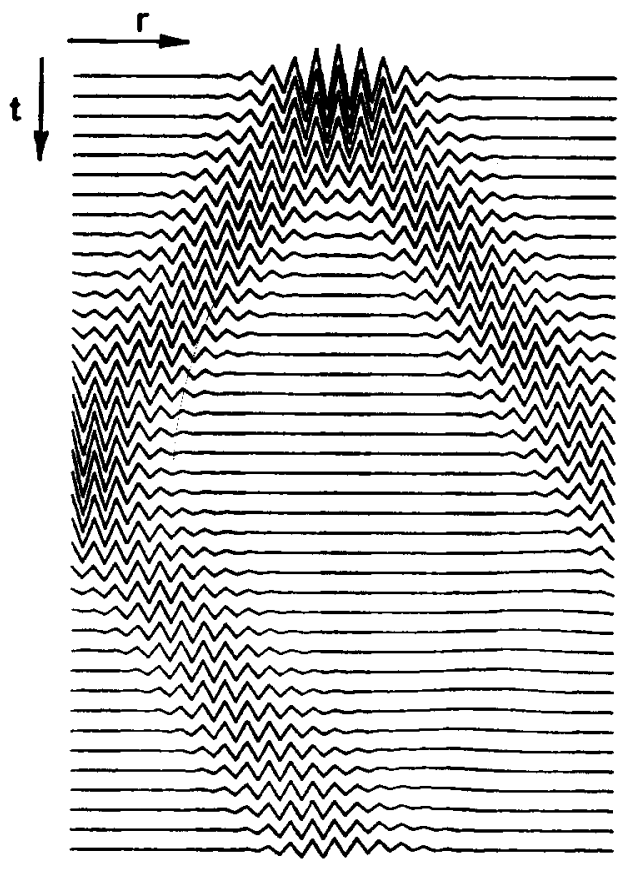

(a)

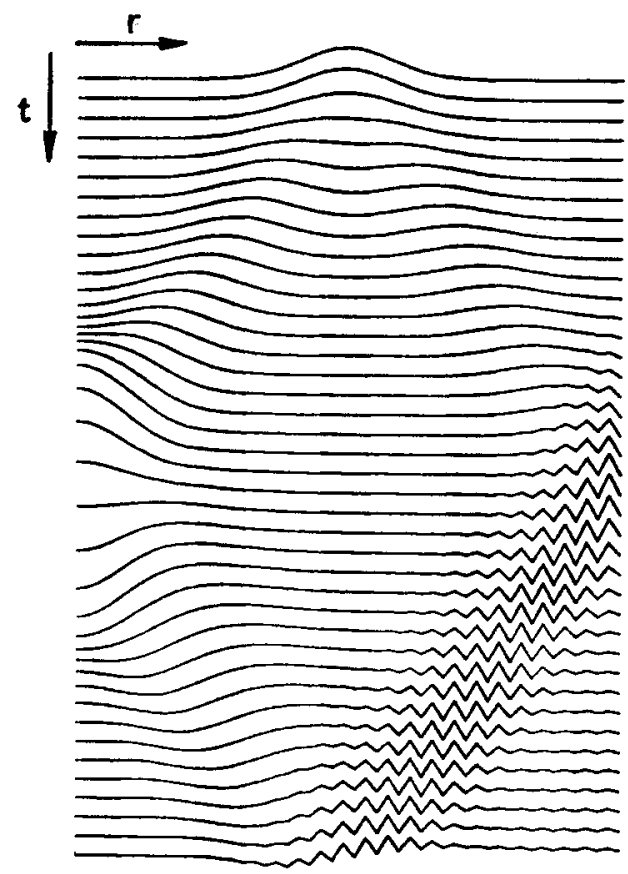

(b)

Figure 7. Axisymmetric linearized Euler equations: (a) absorption of a high-frequency wave and (b) reflection of a lowfrequency pressure wave by BCs of equation (18)

\section{PARTIAL CURES}

The admittance of spurious modes by the $\mathrm{LW}$ scheme lies at the heart of the reflection mechanism, since it is often by the propagation of these modes that errors are carried back from the boundary into the computational domain and corrupt consistent solutions. The natural cure for this spurious behaviour is dissipation, which, unfortunately, tends to diminish in the far field. Any measure that re-establishes dissipation will act towards limiting the damage caused by this spurious behaviour.

\section{Artificial viscosity}

An additional high-order correction term of the general form

$$
\varepsilon\left(u_{j-1}^{n}-2 u_{j}^{n}+u_{j+1}^{n}\right)
$$

is sometimes added to (3) to stabilize the solution in violent flow regions, e.g. near shocks. The coefficient $\varepsilon$ is positive and made data-dependent, e.g.

$$
\varepsilon=\varepsilon_{0} \frac{\left|u_{j+1}-2 u_{j}+u_{j-1}\right|}{\left|u_{j+1}+2 u_{j}+u_{j-1}\right|}
$$

yielding a fourth-order correction term in smooth parts of the flow. Its effect on far-field flows is thus, by construction, very limited, since these flows are very smooth and very slowly varying. 


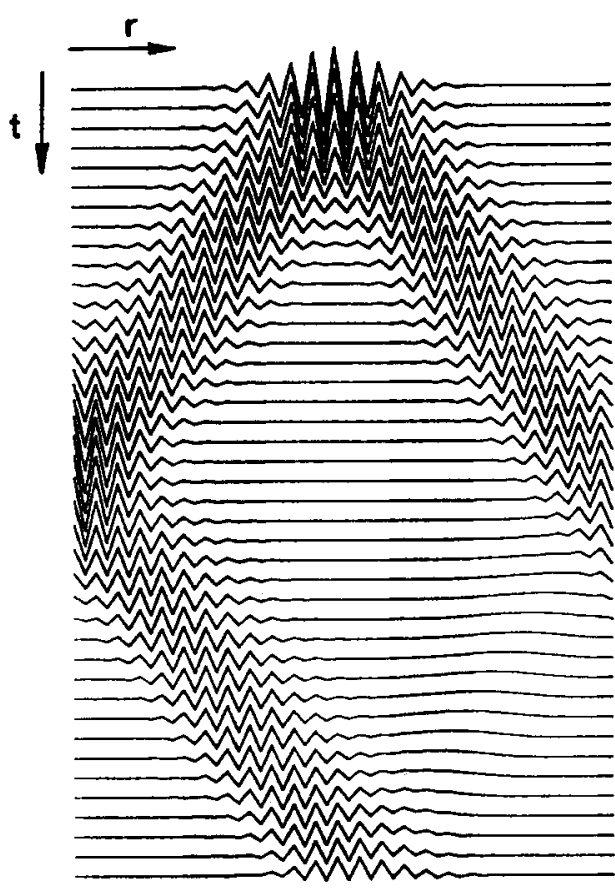

(a)

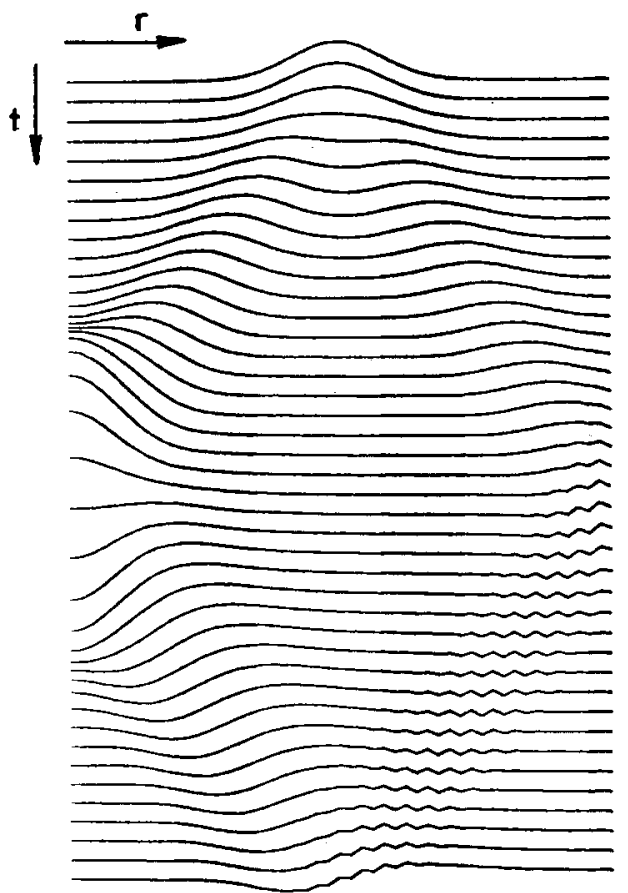

(b)

Figure 8. Axisymmetric linearized Euler equations: absorption of (a) high- and (b) low-frequency pressure waves by combined low-high-frequency BCs (20)

\section{Local time stepping (LTS)}

In computations where steady state solutions are sought by means of integrating the timedependent equations until convergence is reached, the accuracy of transient solutions is sometimes sacrificed for accelerated convergence properties. In an explicit time-marching algorithm the stability constraint on the allowable time step takes the form $|k \lambda / h| \leq C$, where $\lambda$ is the maximal signal speed and $C$ is a scheme-dependent constant. A global restriction on $k$ is usually placed by the smallest cells and is very wasteful on the large cells where information could be allowed to propagate a lot faster. The LTS approach makes $k$ data- and grid-dependent in that it advances the solution by the maximum locally allowable time step $k_{j}$, which varies from one grid cell to another. The transient phase of the solution loses its physical significance but convergence is accelerated. LTS increases local CFL numbers, hence partly compensates for the loss of inherent dissipation due to the coarseness of far-field grids. However, a local stability constraint is placed by the fastest local wave speed $k_{j} \leq C h_{j} /\left|\lambda_{\max }\right|$, and CFL numbers of the other waves, particularly in stiff systems, may still become very small and induce large dispersion errors. LTS, already limited to steady state calculations only, offers no genuine cure to the reflection problem discussed earlier and is of course altogether inapplicable to time-accurate calculations.

\section{Upwinding}

Finally, we mention very briefly that using upwind algorithms may get round the difficulties arising from the admittance of spurious modes. Although upwind models, just like symmetric ones, tend to become non-dissipative in the far field, they still maintain sufficient dissipation levels 
to damp spurious modes over relatively short length scales. ${ }^{16}$ This should come as no complete surprise, since upwind schemes are known for their heavy inherent damping. Precisely this property makes them unsuitable for certain classes of problems where the observed signals are already very weak. Another drawback is that upwind schemes are more expensive since they require simple wave decomposition to ensure stability.

\section{CONCLUDING REMARKS}

We have presented a thorough numerical study of reflections from artificial far-field boundaries and exposed the key numerical parameters upon which absorption level depends. The study concentrated on dissipative models and an LW-type scheme was used as a prototype. The emphasis was on systems where it is the combination of interior scheme and all boundary procedures that determines the absorption efficiency. The numerical tests followed theoretical analysis which suggested that in the far field, local wave numbers are high and induce large dispersion errors. At the same time, local CFL numbers are small and the inherent dissipation of the scheme tends to vanish, allowing reflected spurious modes to persist and corrupt consistent physical solutions. Although shown only for the LW scheme, this last result is more general and applies to a much wider class of symmetric dissipative schemes. ${ }^{16}$ We have presented a variety of examples where strong reflections occur at artificial boundaries, even in 1D and quasi-1D set-ups, cases where theory predicts best results. The conditions required to expose far-field reflections were nowhere as severe as one may encounter in practical calculations. For example, the rate of grid expansion in exterior aeronautical calculations is a lot greater than $5 \%$ and the resulting farfield CFL numbers, particularly in time-accurate calculations, are a lot smaller than the ones used in this study, both of which tend to enhance the reflection mechanism discussed in this work. We have shown internal reflections due to mildly expanding grids and demonstrated strong focusing of asymptotic errors generated at the artificial boundaries. We followed suggestions in References 5 and 7 and designed combined low-high-frequency boundary operators which improved the absorption of both frequency ranges. Finally, we have briefly discussed partial cures, primarily by pointing out their theoretically limited potential and consequently their incapability of offering genuine cures.

\section{ACKNOWLEDGEMENTS}

I would like to thank Professor P. L. Roe for his continuous interest in and advice on this work. I am also grateful to Dr. E. F. Toro for reading a previous version of this manuscript and making valuable remarks.

\section{REFERENCES}

1. B. Engquist and A. Majda, 'Absorbing boundary conditions for the numerical solutions of waves', Math. Comput., 31, 629-651 (1977).

2. A. Bayliss and E. Turkel, 'Radiation boundary conditions for wave like equations', Commun. Pure Appl. Math., 33, 707-725 (1980).

3. G. W. Hedstrom, 'Nonreflecting boundary conditions for nonlinear hyperbolic systems', J. Comput. Phys., 30, 222-237 (1979).

4. P. L. Roe, 'Remote boundary conditions for unsteady multidimensional aerodynamic computations', Comput. Fluids, 17, 221-231 (1989).

5. R. L. Higdon, 'Absorbing boundary conditions for difference approximations to the multi-dimensional wave equation', Math. Comput., 47, 437-459 (1986).

6. E. L. Lindman, 'Free space boundary conditions for time dependent wave equation', J. Comput. Phys., 18, 66-78 (1975).

7. R. Vichnevetsky and E. C. Pariser, 'Non-reflecting upwind boundaries for hyperbolic equations., Numer. Methods $P D E s, 2,1-12$ (1986). 
8. D. H. Rudy and J. C. Strikwerda, 'A nonreflecting outflow boundary condition for subsonic Navier-Stokes calculations', J. Comput. Phys., 36, 55-70 (1980).

9. M. G. Hall, 'Cell vertex multigrid schemes for solutions of the Euler equations', in K. W. Morton and M. J. Baines (eds), Numerical Methods for Fluid Dynamics II, Oxford University Press, Oxford, 1985, pp. 303-345.

10. L. N. Trefethen, 'Group velocity in finite difference schemes', SIAM Rev., 24, 113-136 (1982).

11. R. Vichnevetsky, 'Wave propagation analysis of difference schemes for hyperbolic equations: a review', Int. $j$. numer. methods fluids, 7, 409-452 (1987).

12. M. Giles and W. T. Thompkins Jr., 'Internal reflection due to a nonuniform grid', in R. Vichnevetsky and R. S. Stepleman (eds), Advances in Computational Methods for PDEs V, IMACS, North-Holland, 1984, pp. 322-328.

13. G. B. Witham, Linear and Nonlinear Waves, Wiley-Interscience, New York, 1974.

14. L. N. Trefethen, 'Group velocity interpretation of the stability theory of Gustafsson, Kreiss and Sundström', J. Comput. Phys., 49, 199-217 (1983).

15. A. Iserles, 'Generalized leap frog methods', IMA J. Numer. Anal., 6, 381-392 (1986).

16. S. Karni, 'On the group velocity of symmetric and upwind schemes', Preprint, 1989, to appear.

17. A. Jameson, W. Schmidt and E. Turkel, 'Numerical solutions of the Euler equations by finite volume methods using Runge-Kutta time stepping schemes', AIAA paper 81-1259 (1981).

18. E. Tadmor, 'The unconditional instability of inflow-dependent boundary conditions in difference approximations to hyperbolic systems', Math. Comput., 41, 309-319 (1983).

19. P. L. Roe, 'Approximate Riemann solvers, parameter vectors and difference schemes', J. Comput. Phys., 43, 357-372 (1981).

20. S. Karni, 'Far field boundaries and their numerical treatment', Ph.D. Thesis, College of Aeronautics, Cranfield Institute of Technology, 1990. 